\title{
Pretreatment of Rice Straw using Deep Eutectic Solvent and Saccharification of Pretreated Residue by Crude Cellulase Enzyme
}

\author{
Poonam Maan* and R. S. Sengar
}

Department of Agriculture Biotechnology, College of Agriculture, Sardar Vallabhbhai Patel

University of Agriculture and Technology, Meerut-250110, India

*Corresponding author

\section{Keywords \\ Woody biomass, Agricultural residues, Rice straw, Lignin extraction, Fossil fuel}

Article Info

\section{Accepted:}

15 September 2019

Available Online:

10 October 2019

\section{Introduction}

With the increasing world's population, energy consumption has increased many folds over the last century. Fossil fuel has been the major source of energy that formed from fossils over millions of years within the earth. Fossil fuels are considered as nonrenewable and depleting continuously with a predicted estimation from the 25 billion barrels to approximately 5 billion barrels till 2050 (Campbell and Laherrere, 1998). On the other hand, a large amount of agro-industrial wastes and crop residues are generated all over the globe and creates many environmental problems if not utilized properly. Mainly three categories of lignocellulosic biomass; woody biomass, agricultural residues, and energy crops are produced. Among the agricultural residues, rice straw is generated in abundance (667.6 million tons) in Asian countries every year and would serve as a great potential feedstock for biofuel production. After harvesting the rice, large amount of rice straw residues i.e., $40-50 \mathrm{~cm}$ of loose stubble and $50-60 \mathrm{~cm}$ of standing straw are usually left over the field and it is still burnt in the fields causing severe environmental and health issues (Liu et al., 2011; Binodet al., 2010). It is estimated that 
approximately 205 billion liters of bioethanol can potentially be produced annually by using rice straw (Belal, 2013). Rice straw consists of mainly cellulose (32-47 \%), hemicellulose (19-27\%), and lignin (5-24\%).

Therefore, considering the above problems, efforts have been concentrated in exploring the alternative energy sources such as biofuels (biodiesel and bioethanol) by utilizing lignocellulosic biomass. The most important biomass processing challenge is the pretreatment for production of biofuels and it should be simple, environmental friendly and economically feasible (Ravindran et al., 2018). Pretreatment is required for loosen the complex structure of lignocellulosic biomass. Moreover, lignocellulosic biomass requires suitable pretreatment method for solubilization, separation and conversion of its cellulose and hemicellulose components into fermentable sugars (Sun et al., 2016). In addition, a successful pretreatment process increased the yields of hydrolysis, reduces the product degradation and reduces the formation of inhibitory byproducts. A list of pretreatment methods have been developed for different lignocellulosic biomass over the past few decades but till now, there is no single pretreatment method available that could suit to all types of biomass. The most commonly used fundamental types of pretreatment technologies for lignocellulosic biomass include usage of common organic solvents. These technologies have many disadvantages such as low yield, high processing cost and create health and environmental issues (Anwar et al., 2014; Alvira et al., 2010). Therefore, a continuous search is constantly on-going towards more novel, cheaper and efficient green technologies over the past Decade, for solving these challenges (Liu et al., 2014; Dai et al., 2013a; Capolupo and Faraco, 2016). Recently, the "Green Chemistry" concept has emerged as a possible solution to the challenge of using nontoxic and environmental friendly materials for efficient utilization of lignocellulosic biomass. Ionic liquids (ILs) and Deep Eutatic Solvents (DES) are currently gaining importance as an alternative to conventional pretreatment technologies of biomass. ILs possesses attractive properties due to their higher thermal and chemical stability, negligible vapor pressure and non-flammability nature (Wahlström and Suurnäkki, 2015; Wu et al., 2014). However, the use of ILs in the pretreatment is environmentally friendly but it is a cost-intensive process; this limits its use in biorefinery (Hou et al., 2013). Recently, similar to the ILs, DES attracts the attention as a potential green and designer solvent with several chemical and biological applications (Dai et al., 2013a, b; Choi et al., 2011; Huang et al., 2013). According to Paiva et al., (2014), the yield of the DES preparation process may be considered $100 \%$ as no chemical reaction takes place in its production and total waste production is zero.DES is a mixture of a hydrogen-bond acceptor and donor; in most cases, a quaternary ammonium halide salt act as a hydrogen-bond acceptor and amino acid, urea, amine, carboxylic acid or carbohydrate etc. act as a hydrogen-bond donor (Francisco et al., 2012; Zhang et al., 2012). DES has been used extensively in the recent past years in the pretreatment of lignocellulosic biomass for achieving high hydrolysis and fermentation yields. In the current investigation, the pretreatment of rice straw through acid, alkali and DES (ChCl/Urea) and enzymatic hydrolysis for fermentable sugar production were reported.

\section{Materials and Methods}

\section{Microorganism and enzyme production}

The microorganism used in this study for cellulase enzyme production, was isolated from decomposing wood samples and identified as $C$. cinereaRM-1 NFCCI-3086 by 
National Fungal Culture Collection of India, Agharkar Research Institute, Pune, India. Growth conditions of $C$. cinerea RM-1 for cellulase enzyme production were set as described by Poonam (2015).

\section{Raw material and chemicals}

Rice straw was procured from the local rice field and washed 3-4 times in distilled water, dried and powdered using laboratory grinder, dried again and stored in polythene begs. This rice straw was used as lignocellulosic biomass for pretreatment studies. Carboxymethyl cellulose (CMC), birchwoodxylan, glucose, xylose, and arabinose were purchased from Sigma. All other chemicals and reagents were of analytical grade.

\section{DES preparation and rice straw pretreatment}

DES reagent was prepared in capped bottle by mixing $\mathrm{ChCl} /$ Urea at a molar ratio of $2: 1$ and incubated in incubation shaker at $100 \mathrm{rpm}$ and $70{ }^{\circ} \mathrm{C}$ until a clear liquid solution was obtained (Dai et al., 2013a, Francisco et al., 2013b). Various pretreatment experiments including, $2 \% \mathrm{H}_{2} \mathrm{SO}_{4}, 2 \% \mathrm{NaOH}$ and DES solvent $(\mathrm{ChCl} / \mathrm{Urea})$ were carried out in screw capped conical flasks at $10 \%$ solid loadings unless mentioned. Briefly, $10 \mathrm{~g}$ of rice straw was mixed with $2 \% \mathrm{H}_{2} \mathrm{SO}_{4}, 2 \% \mathrm{NaOH}$ and DES solvent in a solid/liquid ratio of 1:10 separately, and subjected to steam treatment at $121{ }^{\circ} \mathrm{C}$ and 15 psi pressure for $30 \mathrm{~min}$. Following this, the samples were washed with distilled water for three times. Control and pretreated rice straw samples were analyzed for cellulose, hemicellulose and lignin content.

\section{Lignin separation and recovery of DES}

DES was separated from lignin by adding distilled water until the turbid solution obtained. The solution was incubated at $5{ }^{\circ} \mathrm{C}$ for 3h. After incubation, the mixture was centrifuged at $10000 \times \mathrm{g}$ for $15 \mathrm{~min}$, and the pellet was washed with distilled water three times and air-dried to obtain lignin powder. DES was recovered from water solution by incubation in vacuum rotary evaporator at 60 ${ }^{\circ} \mathrm{C}$. The recovered DES and pure water may be reused in the next biomass pretreatment and lignin precipitation cycle.

\section{Solubility evaluation test of cellulose, xylan, and lignin in DES}

Pure cellulose, xylan, and lignin were used for Specific solubility test in DES reagents. All three components were dissolved in $10 \mathrm{ml}$ $\mathrm{ChCl} /$ Urea at a molar ratio of $2: 1$ and $3: 1$ separately in $10 \%$ concentration and incubated at $60{ }^{\circ} \mathrm{C}$ for $12 \mathrm{~h}$. All samples were filtered through fiber glass filters and dried at $60{ }^{\circ} \mathrm{C}$. The solubility (\%) of the NADES reagent was determined by calculating the weight of dried components.

\section{Enzymatic hydrolysis of pretreated rice straw}

Enzymatic scarification experiments were carried out at $10 \%$ solid loading and cellulase enzyme dose of $10 \mathrm{IU}$ per $\mathrm{g}$ in a total reaction volume of $10-\mathrm{ml}$ with citrate buffer in $50-\mathrm{ml}$ sealed bottles. The prepared mixtures were then incubated at $45{ }^{\circ} \mathrm{C}$ and $15 \mathrm{rpm}$ for 24 and $48 \mathrm{~h}$.

The reducing sugars were measured by DNS method (Miller, 1959).The Control experiments were carried out separately either by avoiding cellulose enzyme or the pretreated substrate.

\section{Analytical methods}

The cellulase activity (CMCase) was determined using $\mathrm{CMC}$ as the substrate following the protocol published by Mandels 
M. (1975). The compositional analysis of raw and pretreated straw was done for determining the cellulose, hemicellulose, and lignin content (Sluiter et al., 2008).

\section{Results and Discussion}

\section{Pretreatment of rice straw}

Table 1 represents the effects of different solvents i.e., dilute acid, mild alkali and DES on delignification of rice straw. Acid treatment of rice straw has very high detrimental effect on hemicellulose content and it decreased from $24.6 \%$ to $1.2 \%$ while the cellulose and lignin content are not affected to much extent. After alkali pretreatment, the overall cellulose content increased from $35.5 \%$ to $43.8 \%$ in pretreated rice straw while hemicellulose and lignin content decreased from $24.6 \%$ to $18.5 \%$ and from $14.5 \%$ to $11.56 \%$, respectively. DES pretreatment of rice straw resulted in decrease in lignin content from $14.5 \%$ to $8.7 \%$, while cellulose content was increased from $35.5 \%$ to $45.8 \%$ and hemicellulose content decreased from $24.6 \%$ to $21.5 \%$. Thus, it is observed that lignin content was decreased about $40 \%$ without affecting the hemicellulose very much after DES pretreatment; thus the overall amount of cellulose was enhanced. Generally, acid pretreated biomass showed significant loss in hemicellulose content (Hendriks and Zeeman,2009). While, DES pretreatment showed no severe effect on hemicellulose and cellulose content of biomass. This trend may found because choline chloride stabilizes the cellulose by making hydrogen bond with it; thus, dissolution of cellulose and hemicellulose is inhibited (Abbott et al., 2006). The cellulose content enhancement after pretreatment may be due to the alteration in biomass structure and crystalline cellulose which might have increased the overall cellulose availability (Kumar and Parikh, 2015). Kandanelli et al., (2018) reported the removal of about $50 \%$ lignin from lignocellulosic biomass by using $n$-butanol assisted DES (ChCl: OA) at solid loading of $15 \%(w / v)$ at $120^{\circ} \mathrm{C}$ for $60 \mathrm{~min}$. Kumar et al., (2016) reported that approximately 58\% lignin was removed from rice straw after pretreatment with NADES.

Table 2 represents the solubilization studies of pure cellulose, hemicellulose and lignin in DES solution. The pure cellulose and hemicellulose showed no solubilization in DES and remained untouched while lignin showed high solubility (78\%); this proves the specificity of DES towards lignin solubilization. While, the $\%$ solubility of lignin when present in biomass was found to be comparatively lower than the pure lignin in DES. This could be due to the cross-linking architecture of biomass and strong binding of lignin to cellulose and hemicellulose which poses the lignin to extract. These finding were in close agreement with Kroon et al., (2014), who reported that NADES showed very high selectivity for separation of lignin from a mixture of lignin and cellulose and that lignin solubility values varied with different combinations of NADES reagents.

\section{Saccharification studies}

Table 3 represents the enzymatic hydrolysis results of rice straw pretreated with DES at $10 \%$ solids loading and $10 \mathrm{IU} / \mathrm{g}$ of crude cellulase enzyme produced by $C$. cinerea. The hydrolysis experiments were performed at 45 ${ }^{\circ} \mathrm{C}$ and $15 \mathrm{rpm}$.

The reducing sugars were measured after 24 and $48 \mathrm{~h}$. The results showed the maximum saccharification efficiency of $31.5 \pm 2.5 \%$ with reducing sugar yields of $385 \pm 8 \mathrm{mg} / \mathrm{g}$ after 48 h. Our results were in line with Kumar et al., (2015) who reported that enzymatic hydrolysis of rice straw showed reducing sugars yield of $333 \pm 11 \mathrm{mg} \mathrm{g}-1$ and saccharification efficiency of $36.0 \pm 3.2 \%$ in $24 \mathrm{~h}$ at $10 \%$ solids loading. 
Table.1 Compositional analysis of raw and pretreated rice straw

\begin{tabular}{|c|c|c|c|c|}
\hline S. No. & Treatment & Cellulose\% & Hemicellulose\% & Lignin\% \\
\hline 1. & Untreated rice straw & $35.5 \pm 1.2$ & $24.6 \pm 1.2$ & $14.5 \pm 1.1$ \\
\hline 2. & Acid treated rice straw & $34.2 \pm 2.2$ & $1.2 \pm 0.8$ & $13.6 \pm 0.9$ \\
\hline 3. & Alkali treated rice & $43.8 \pm 3.4$ & $18.52 \pm 1.1$ & $11.56 \pm 0.9$ \\
& straw & & & \\
\hline 4. & DES treated rice straw & $45.8 \pm 2.7$ & $21.5 \pm 1.7$ & $8.7 \pm 0.4$ \\
\hline
\end{tabular}

Table.2 Solubility analysis of pure cellulose, xylan, and lignin in (ChCl/Urea)

\begin{tabular}{|c|c|c|c|}
\hline \multirow{2}{*}{ DES reagent } & \multicolumn{3}{|c|}{$\%$ Solubility (10\% (w/v) } \\
& Substrate) \\
\cline { 2 - 4 } & Cellulose & Xylan & Lignin \\
\hline $\begin{array}{c}\text { ChCl/Urea } \\
(\mathbf{2 : 1 )}\end{array}$ & 0.0 & 0.0 & $75.1 \pm 6$ \\
\hline $\begin{array}{c}\text { ChCl/Urea } \\
(\mathbf{3 : 1 )}\end{array}$ & 0.0 & 0.0 & $73.5 \pm 5.5$ \\
\hline
\end{tabular}

Table.3 Enzymatic saccharification of DES pretreated rice straw biomass

\begin{tabular}{|c|c|c|}
\hline Time of Saccharification & Reducing sugars $(\mathbf{m g} / \mathbf{g})$ & $\begin{array}{c}\text { Saccharification efficiency } \\
(\mathbf{\%})\end{array}$ \\
\hline $\mathbf{2 4 h}$ & $282 \pm 10$ & $20 \pm 1.2$ \\
\hline $\mathbf{4 8 h}$ & $385 \pm 8$ & $31.5 \pm 2.5$ \\
\hline
\end{tabular}

Here, we have revealed a green pretreatment process for biomass using deep eutectic solvent $(\mathrm{ChCl} / \mathrm{Urea})$ as a potential extraction media for delignification from rice straw and compared with acid and alkali treatment.

A high-quality lignin was extracted from biomass and was separated from cellulose and hemicellulose in a single step by simple precipitation method.

The Results showed that approximately $40 \%$ lignin were removed from rice straw using DES treatment. Following delignification, the residual rice straw was subjected to enzymatic hydrolysis and a plenty amount of fermentable sugars $(385 \pm 8 \mathrm{mg} / \mathrm{g})$ were produced with a saccharification efficiency of $36.0 \pm 3.2$ $\%$.After pretreatment with DES, degradation products such as furfural and hydroxyl methyl furfural are not formed; therefore detoxification step is not required which is a key step after acid pretreatment.

Therefore, DES pretreatment decreases the overall cost of process by reducing the postprocess steps and these green solvents may absolutely be used as the next-generation reagents for sustainable development.

\section{Acknowledgement}

The authors are thankful to Sardar Vallabhbhai Patel University of Agriculture \&Technology, Meerut for providing the lab facilities. The authors acknowledge the financial support from University Grants Commission, New Delhi, India. 


\section{References}

Abbott, A.P., Bell, T.J., Handa, S., and Stoddart, B. 2006. Cationic functionalization of cellulose using a choline based ionic liquid analogue. Green Chem 8:784-786.

Alvira, P., Tomas-Pejo, M., Ballesteros, M., and Negro, M.J. 2010. Pretreatment technologies for an efficient bioethanol production process based on enzymatic hydrolysis: a review. Bioresour Technol 101:4851-4861

Anwar, Z., Gulfraz, M., and Irshad, M. 2014. Agro-industrial lignocellulosic biomass a key to unlock the future bioenergy: a brief review. J Radiat Res ApplSci 7(2):163-173

Belal, B.E. 2013. Bioethanol production from rice straw residues. Braz J Microbiol 44:225-234

Binod, P., Sindhu, R., Singhania, R.R., Vikram, S., Devi, L., Nagalakshmi, S., Kurien, N., Sukumaran, R.K. and Pandey, A. 2010. Bioethanol production from rice straw: an overview. BioresourTechnol 101:47674774.

Campbell, C.J., and Laherrère, J.H. 1998. The end of cheap oil. Scientific American, 278(3), 78-83.

Capolupo, L., and Faraco, V. 2016. Green methods of lignocellulose pretreatment for biorefinery development. Appl. Microbiol. Biotechnol. 100:9451-9467.

Choi, Y.H., Spronsen, J.V., Dai, Y., Verberne, M., Hollmann, F., Arends, IWCE, Witkamp, G.J. and Verpoorte, R. 2011. Are natural deep eutectic solvents the missing link in understanding cellular metabolism and physiology. Plant Physiol. 156:1710-1705.

Dai, Y., Spronsen, J.V., Witkamp, G.J., Verpoorte, R., and Choi, Y.H. $2013 \mathrm{a}$. Natural deep eutectic solvents as new potential media for green technology. Anal ChimActa. 766:61-68.

Dai, Y.,Witkamp, G.J., Verpoorte, R., and Choi, Y.H. 2013b. Natural deep eutectic solvents as a new extraction media for phenolic metabolites in Carthamus tinctorius L. Anal Chem. 85:6272-6278.

Francisco, M., van den Bruinhorst, A., and Kroon, M.C. 2012. New natural and renewable low transition temperature mixtures (LTTMs): screening as solvents for lignocellulosic biomass processing. Green Chemistry. 14(8): 21532157.

Francisco, M., van den Bruinhorst, A., Zubeir, L.F., Peter, C.J., and Kroon, M.C. 2013b. A new low transition temperature mixture (LTTM) formed by choline chloride+lactic acid: characterization as solvent for $\mathrm{CO} 2$ capture. Fluid Phase Equilib. 340:77-84

Hendriks A.T.W.M., and Zeeman, G. 2009. Pretreatments to enhance the digestibility of lignocellulosic biomass. Bioresour Technol. 100:10-18

Hou, X.D., Li, N., and Zong, M.H. 2013. Facile and Simple Pretreatment of Sugar Cane Bagasse without Size Reduction Using Renewable Ionic Liquids-Water Mixtures. ACS Sustainable Chemistry \& Engineering. 1(5):519-526.

Huang, Z.L., Wu, B.P., Wen, Q., Yang, T.X., and Yang, Z. 2013. Deep eutectic solvents can be viable enzyme activators and stabilizers. J ChemTechnolBiotechnol. doi:10.1002/jctb.4285.

Kandanelli, R., Thulluri, C., Mangala, R., Rao, P.V., Gandham, S., and Velankar, H.R. 2018. A novel ternary combination of deep eutectic solvent-alcohol (DES-OL) system for synergistic and efficient delignification of biomass. Bioresour. technol. 265:573576.

Kroon, M.C., Casal, M.F., and VandenBruinhorst, A. 2014. Pretreatment of lignocellulosic biomass and recovery of substituents using natural deep eutectic solvents/compound mixtures with low transition temperatures. International Patent. Publication Number: WO 2013/153203 A1.

Kroon, M.C., Casal, M.F., and VandenBruinhorst, A. 2014. Pretreatment of lignocellulosic biomass and recovery of substituents using natural deep eutectic 
solvents/compound mixtures with low transition temperatures. International Patent. Publication Number: WO 2013/153203 A1

Kumar, A.K., and Parikh, B.J. 2015. Cellulose degrading enzymes from Aspergillus terreus D34 and enzymatic saccharification of mildalkali and dilute-acid pretreated lignocellulosic biomass residues. Bioresour Bioprocess. doi:10.1186/s40643-015-00388.

Kumar, A.K., Parikh, B.S., and Pravakar, M. 2016. Natural deep eutectic solvent mediated pretreatment of rice straw: bioanalytical characterization of lignin extract and enzymatic hydrolysis of pretreated biomass residue. Environmental Science and Pollution Research. 23(10):9265-9275.

Liu, Y.T., Chen, Y.A., and Xing, Y.J. 2014. Synthesis and characterization of novel ternary deep eutectic solvents. Chin ChemLett. 25:104-106.

Liu, Z., Xu, A., and Zhao, T. 2011. Energy from combustion of rice straw: status and challenges to china. Energy Power Eng. 3:325-331.

Maan, P., Bharti, A.K., Gautam, S., and Dutt, D. 2016. Screening of important factors for xylanase and cellulase production from the fungus $C$. cinerea RM-1 NFCCI-3086 through plackett-burman experimental design. BioResources, 11(4):8269-8276.

Mandels, M. 1975. Microbial sources of cellulases, Biotech. Bioengg. Symp., 5:81105.

Miller, G.L. 1959.Use of dinitrosalicylic acid reagent for determination of reducing sugar. Anal. Chem. 31:426-428.
Paiva, A., Craveiro, R., Aroso, I., Martins, M., and Reis, R.L. 2014. Natural deep eutectic solvents - Solvents for the 21st century. ACS Sustainable Chem Eng. 2:1063-1071.

Poonam 2015. "Studies on enzymatic aspects of microbial origin and bio-bleaching of hardwoods," Ph.D. Dissertation, Indian Institute of Technology, Roorkee, India.

Ravindran, R., Jaiswal, S., Abu-Ghannam, N., and Jaiswal, A.K. 2018. A comparative analysis of pretreatment strategies on the properties and hydrolysis of brewers' spent grain. Bioresour. technol. 248:272-279.

Sluiter, A., Hames, B., Ruiz, R., Scarlata, C., Sluiter, J., Templeton, D., and Crocker, D. 2008. Determination of Structural Carbohydrates and Lignin in Biomass, National Renewable Energy Laboratory, NREL/TP-510-42618.

Sun, S., Sun, S., Cao, X., and Sun, R. 2016. The role of pretreatment in improving the enzymatic hydrolysis of lignocellulosic materials. Bioresour. Technol. 199:49-58.

Wahlström, R.M., and Suurnäkki, A. 2015. Enzymatic hydrolysis of lignocellulosic polysaccharides in the presence of ionic liquids. Green Chem. 17(2):694-714.

Wu, W., Wang, Z., Jin, Y., Matsumoto, Y., and Zhai, H. 2014. Effects of $\mathrm{LiCl} / \mathrm{DMSO}$ dissolution and enzymatic hydrolysis on the chemical composition and lignin structure of rice straw. Biomass and Bioenergy.71:357-362.

Zhang, Q., Vigier, K.D.O., Royer, S. and Jérôme, F., 2012. Deep eutectic solvents: syntheses, properties and applications. Chem. Soc. Rev. 41:7108-7146.

\section{How to cite this article:}

Poonam Maan and Sengar, R. S. 2019. Pretreatment of Rice Straw using Deep Eutectic Solvent and Saccharification of Pretreated Residue by Crude Cellulase Enzyme. Int.J.Curr.Microbiol.App.Sci. 8(10): 1812-1818. doi: https://doi.org/10.20546/ijcmas.2019.810.210 